\title{
Linear FMCW Laser Radar for Precision Range and Vector Velocity Measurements
}

\author{
Diego Pierrottet ${ }^{\mathrm{a}}$, Farzin Amzajerdian ${ }^{\mathrm{b}}$, Larry Petway ${ }^{\mathrm{b}}$, Bruce Barnes ${ }^{\mathrm{b}}$, George Lockard ${ }^{\mathrm{b}}$, and \\ Manuel Rubio ${ }^{\mathrm{b}}$ \\ ${ }^{\mathrm{a} C}$ Coherent Applications, Inc., Hampton VA, 23669 \\ ${ }^{\mathrm{b}}$ NASA Langley Research Center, Hampton VA 23681
}

\begin{abstract}
An all fiber linear frequency modulated continuous wave (FMCW) coherent laser radar system is under development with a goal to aide NASA's new Space Exploration initiative for manned and robotic missions to the Moon and Mars. By employing a combination of optical heterodyne and linear frequency modulation techniques [1-3] and utilizing state-of-the-art fiber optic technologies, highly efficient, compact and reliable laser radar suitable for operation in a space environment is being developed. Linear FMCW lidar has the capability of high-resolution range measurements, and when configured into a multi-channel receiver system it has the capability of obtaining high precision horizontal and vertical velocity measurements. Precision range and vector velocity data are beneficial to navigating planetary landing pods to the preselected site and achieving autonomous, safe soft-landing.

The all-fiber coherent laser radar has several important advantages over more conventional pulsed laser altimeters or range finders. One of the advantages of the coherent laser radar is its ability to measure directly the platform velocity by extracting the Doppler shift generated from the motion, as opposed to time of flight range finders where terrain features such as hills, cliffs, or slopes add error to the velocity measurement. Doppler measurements are about two orders of magnitude more accurate than the velocity estimates obtained by pulsed laser altimeters [4]. In addition, most of the components of the device are efficient and reliable commercial off-the-shelf fiber optic telecommunication components. This paper discusses the design and performance of a second-generation brassboard system under development at NASA Langley Research Center as part of the Autonomous Landing and Hazard Avoidance (ALHAT) project.
\end{abstract}

\section{INTRODUCTION}

The motivation behind the development of an all-fiber high-resolution coherent lidar system comes from the need to meet requirements set by NASA's space exploration initiative. To support activities related to planetary exploration missions, this effort addresses the call for advancement of entry, descent, and landing technologies. Future exploratory missions to the Moon and Mars will become more focused towards landing at locations with high scientific value. This may include targeting sites near cliffs, valleys, craters, or other geographically interesting terrain [5,6]. Exploring technologies that will lead to an efficient and rugged method that provides feedback to the navigation and terrain hazard avoidance systems for soft landing at the targeted landing site are the main goals of this investigation [7]. This paper presents the 
work in progress of an all fiber lidar system capable of providing critical descent range and velocity of the planetary vehicles with a high degree of precision. A brassboard system designed to fly aboard a helicopter is currently under development for a scheduled flight test in August of 2008 over the California desert.

\section{LASER WAVEFORM DESCRIPTION}

The lidar obtains high-resolution range and velocity information from a frequency modulated-continuous wave (FMCW) laser beam whose instantaneous frequency varies linearly with time. Figure 1 shows the transmitted (green) and received (blue) linearly chirped triangular modulation function.

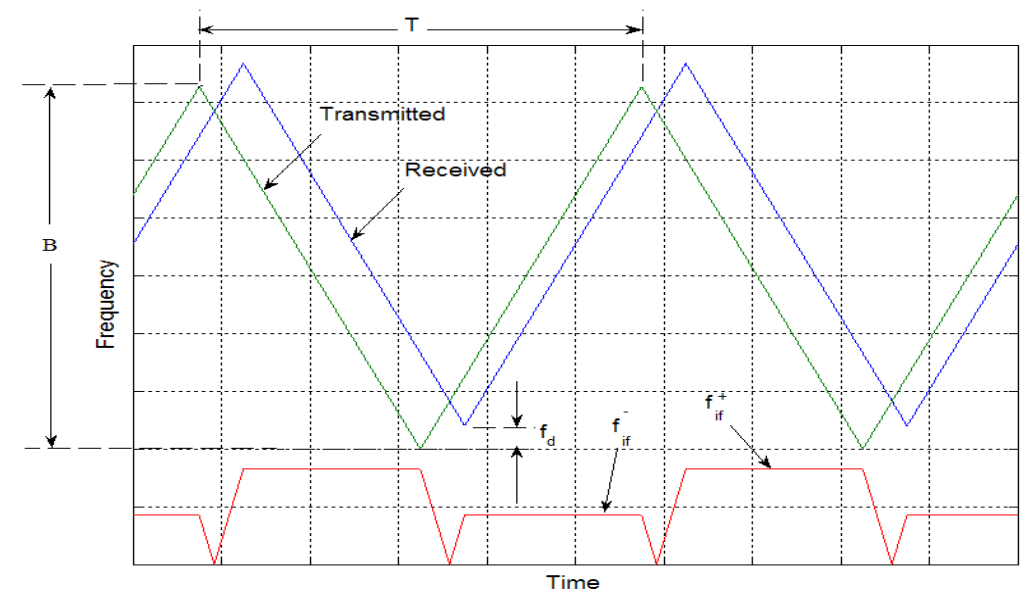

Figure 1. Frequency content of the transmitted and received linear FM-CW waveforms and the resulting time varying IF of the homodyne signal.

In a homodyne configuration, a portion of the transmitted beam serves as the local oscillator (LO) for the optical receiver. Mixing the LO field with the time delayed received field at the detector yields a time varying intermediate frequency (IF) as shown by the red trace in figure 1 , and which is directly related to the target range by the equation

$$
f_{i f}=\frac{4 R B}{T C}
$$

where $\mathrm{R}$ is the range to target, $\mathrm{B}$ is the modulation bandwidth, $\mathrm{T}$ is the waveform period and $\mathrm{c}$ is the speed of light. For the case of a moving target, a Doppler frequency shift will be superimposed to the IF, that is a change in frequency over the waveform up-ramp $\left(\mathrm{f}_{\mathrm{if}}{ }^{+}=\mathrm{f}_{\mathrm{if}}+\mathrm{f}_{\mathrm{d}}\right)$ and a decrease during down-ramp $\left(f_{i f}=f_{i f}-f_{d}\right)$. Therefore in presence of a Doppler shift, the frequency $f_{\text {if }}$ used in equation 1 for determining the target range is replaced by

$$
f_{i f}=\frac{f_{i f}^{+}+f_{i f}^{-}}{2}
$$

The Doppler frequency shift for the case when the shift is less than $f_{\text {if }}$ is simply 


$$
f_{d}=\frac{f_{i f}^{+}-f_{i f}^{-}}{2}
$$

Target radial velocity component is obtained from the equation

$$
v=\frac{f_{d} \lambda}{2 \cos \theta}
$$

where $\lambda$ is the transmitter laser wavelength, and $\theta$ is the angle between the target velocity vector and the lidar line of sight.

Horizontal and vertical vector velocity components are obtained by dividing the transmit laser power into three beams and angularly separating each laser beam from each other by 120 degrees in azimuth, and approximately 45 degrees in nadir. Having prior knowledge of the orientation of the beams relative to the platform direction of motion allows us to measure horizontal as well as vertical velocities without ambiguities. In addition, since there are still two solutions for each set of horizontal and vertical frequency components the sensor can distinguish between positive or negative Doppler frequency shifts by keeping track of the waveform's ramp up and ramp down temporal locations. For example, consider the case of a positive Doppler shift during forward motion; the IF between the received signal and the LO during the up-ramp will be less than the IF during the down ramp as is displayed in figure 1. If the motion is in the opposite direction, the up-ramp IF will be greater than the down-ramp IF. By comparing the IF of the up-ramp to the IF of the down-ramp, the Doppler ambiguity is removed for each of the three transmitted beams.

\section{SYSTEM DESCRIPTION}

The performance quality of the device comes primarily from the line width of the seed laser and the linearity of the modulated waveform. Laser line-width limits the IF measurement resolution, and waveform linearity limits signal to noise ratio (SNR) [8, 9]. The system to be installed and field-tested aboard a helicopter uses a frequency stabilized, fiber laser with a spectral line width that is less than $10 \mathrm{KHz}$ full width, at half maximum. A great deal of effort was devoted to achieving a linear modulation and transform limited signal spectra operation. Figure 2 shows the measured laser frequency vs. time illustrating nearly perfect waveform linearity. 


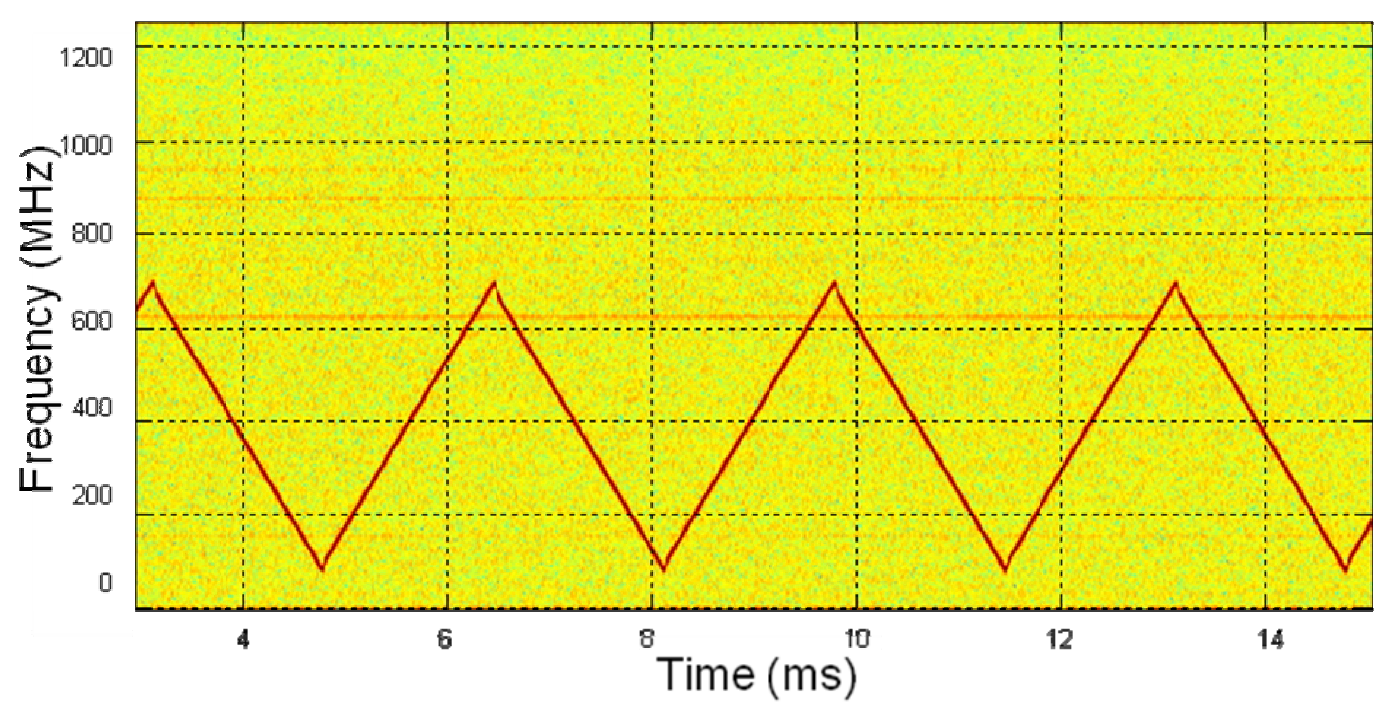

Figure 2. The spectrogram shows the frequency modulated waveform, which was obtained by optically mixing the lidar waveform with a stable continuous wave reference laser.

Figure 3 is a block diagram that illustrates the configuration of the all fiber FMCW lidar sensor. The FMCW waveform generated at the seed laser is amplified by a high power fiber amplifier. Line width measurements at the output of the amplifier show negligible line broadening by the amplifier. The output of the fiber amplifier is split into three components in order to distribute the power to three telescopes at the sensor's optical head. The optical head consists of the fiber to free space coupling telescopes, which can be mounted separately from the rest of the system anywhere on the vehicle platform that has a clear view of the target. The signals from the ground are collected by these telescopes and sent to three pairs of dual balanced detectors via optical fiber cables. The outputs of the detectors are processed by a Pentium Dual Core processor based receiver, capable of either storing the temporal data for post processing, or providing real-time range and velocity measurements that are currently displayed on a graphical user interface (GUI). Real-time range and velocity measurement is a critical capability necessary for precision navigation. All system electronics and fiber optic components are housed in a standard instrumentation rack. 


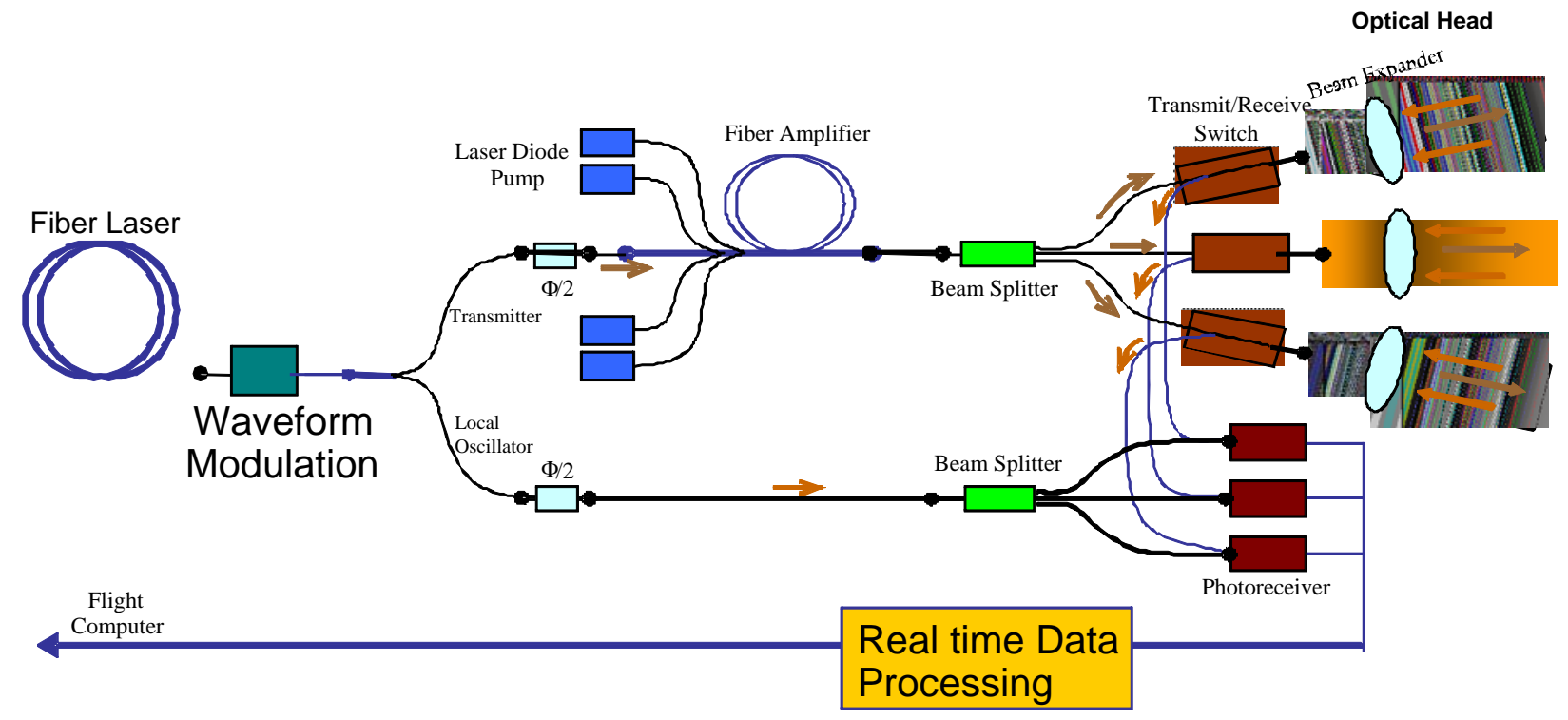

Figure 3. All fiber, coherent Doppler lidar system schematic.

\section{SAMPLE DATA PRODUCTS}

Performance characterization of the lidar is ongoing at the ALHAT Sensor Test Range (STR) at NASA Langley Research Center. The sensor test range includes a target board with diffuse surfaces of known reflection coefficients and different size panels at a 250 meter range [10]. A photograph of the STR is shown in figure 4 for reference. Range characterization experiments of the FMCW lidar were made on the right section of the STR consisting of $30 \mathrm{~cm}$ wide by 2 meter long panels that extend away from the backboard. The distance between the panels and the backboard range from $25 \mathrm{~cm}$ to $5 \mathrm{~cm}$ from top to bottom, in steps of $5 \mathrm{~cm}$ increments, and one panel is set at $2.5 \mathrm{~cm}$ at the bottom for a total of six panels. To test the range measurement performance of the FMCW lidar, the fiber-coupled telescopes are mounted on a rotating stepping motor that scans the laser beam along a vertical path over the target board. Figure 5 shows the ranges calculated from the measured signal IF per equation 1 . Superimposed on the measured data are the actual range values. 


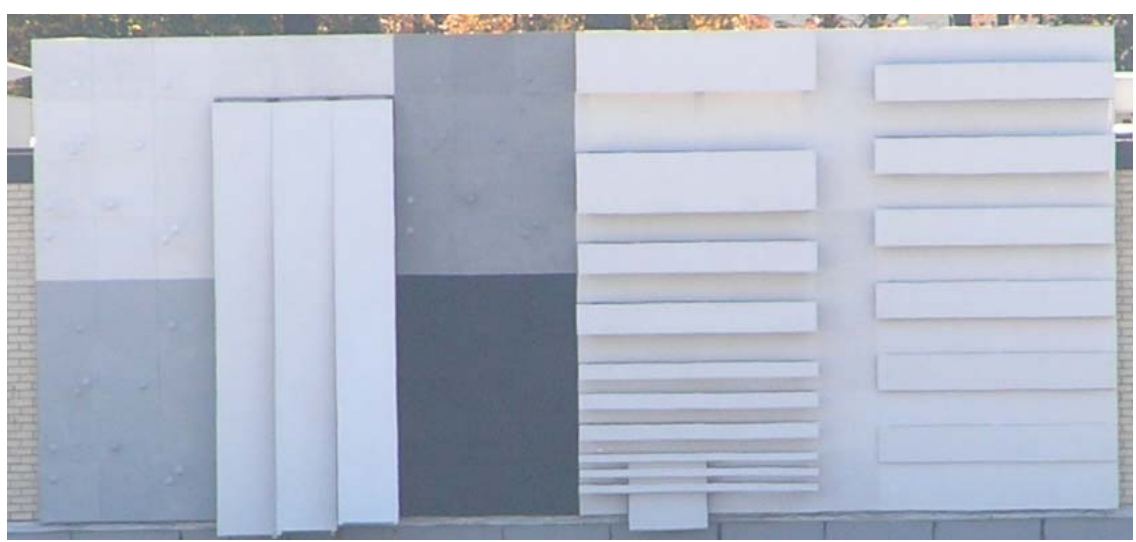

Figure 4. The Sensor Test Range at NASA LaRC

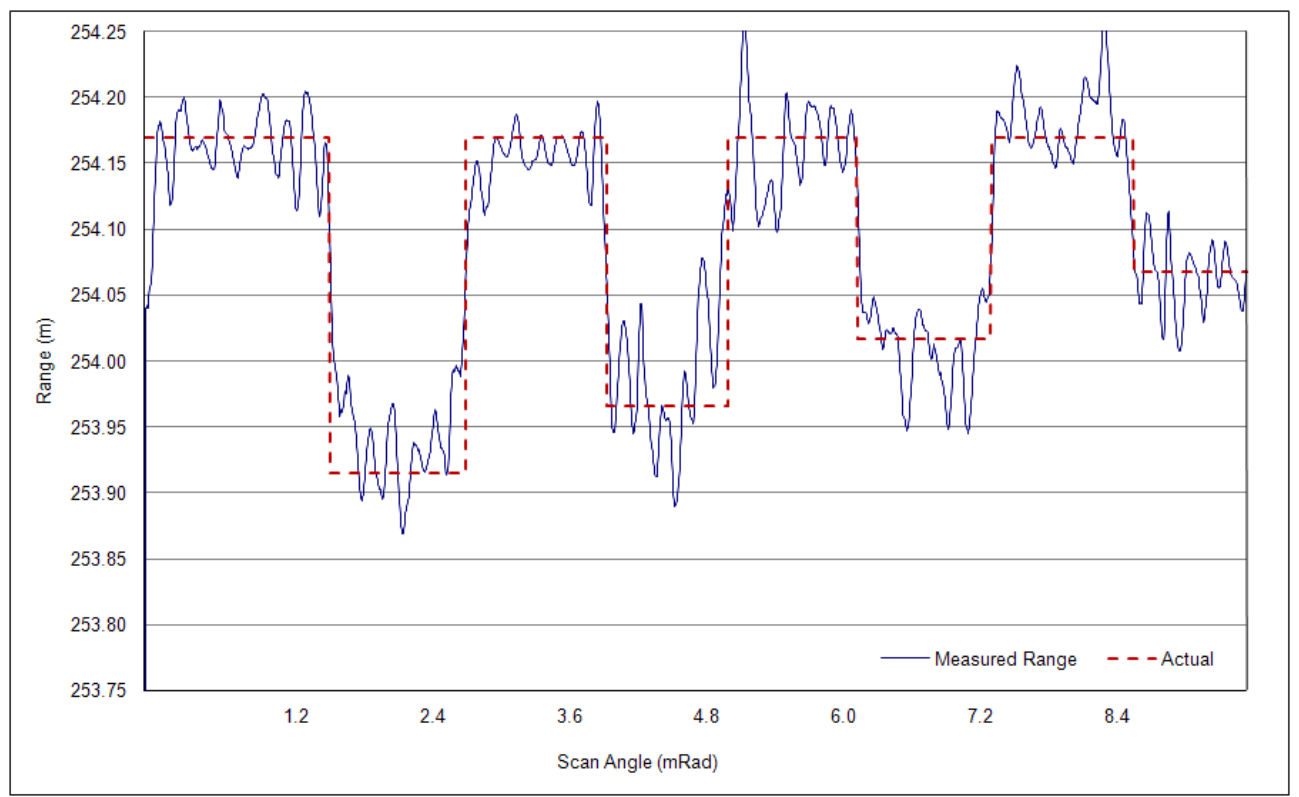

Figure 5. Range measurements obtained from the STR.

The panels shown in figure 5 correspond to the $25 \mathrm{~cm}$ panel at the left, followed by the 20, 15 and down to the $10 \mathrm{~cm}$ panel on the right of the chart. This preliminary data collected by the real-time system shows that the measured range values agree well with the true values to less than $5 \mathrm{~cm}$. Ongoing improvements are expected to improve the range measurement accuracy to about $1 \mathrm{~cm}$.

To generate velocity signals, a rudimentary wheel was constructed and mounted on an electric motor. The perimeter surface of the wheel consists of a rough sandy texture uniformly painted with a color of known diffuse reflectance at the lidar wavelength. The wheel has a 91 $\mathrm{cm}$ diameter, and a perimeter surface width of $15 \mathrm{~cm}$. Figure 6 shows the measured velocity with respect to time at a position on the wheel that is approximately 45 degrees up from normal 
incidence (normal incidence is the line of sight angle pointed to rotation axis of the wheel). As the data was collected, velocity variations were detected and displayed by the real-time data acquisition and processing system. The periodic variations of the target velocity were caused by wheel wobble, and the sudden change in the velocity is due to a wind gust pushing on the wheel, as pointed out in figure 6 .

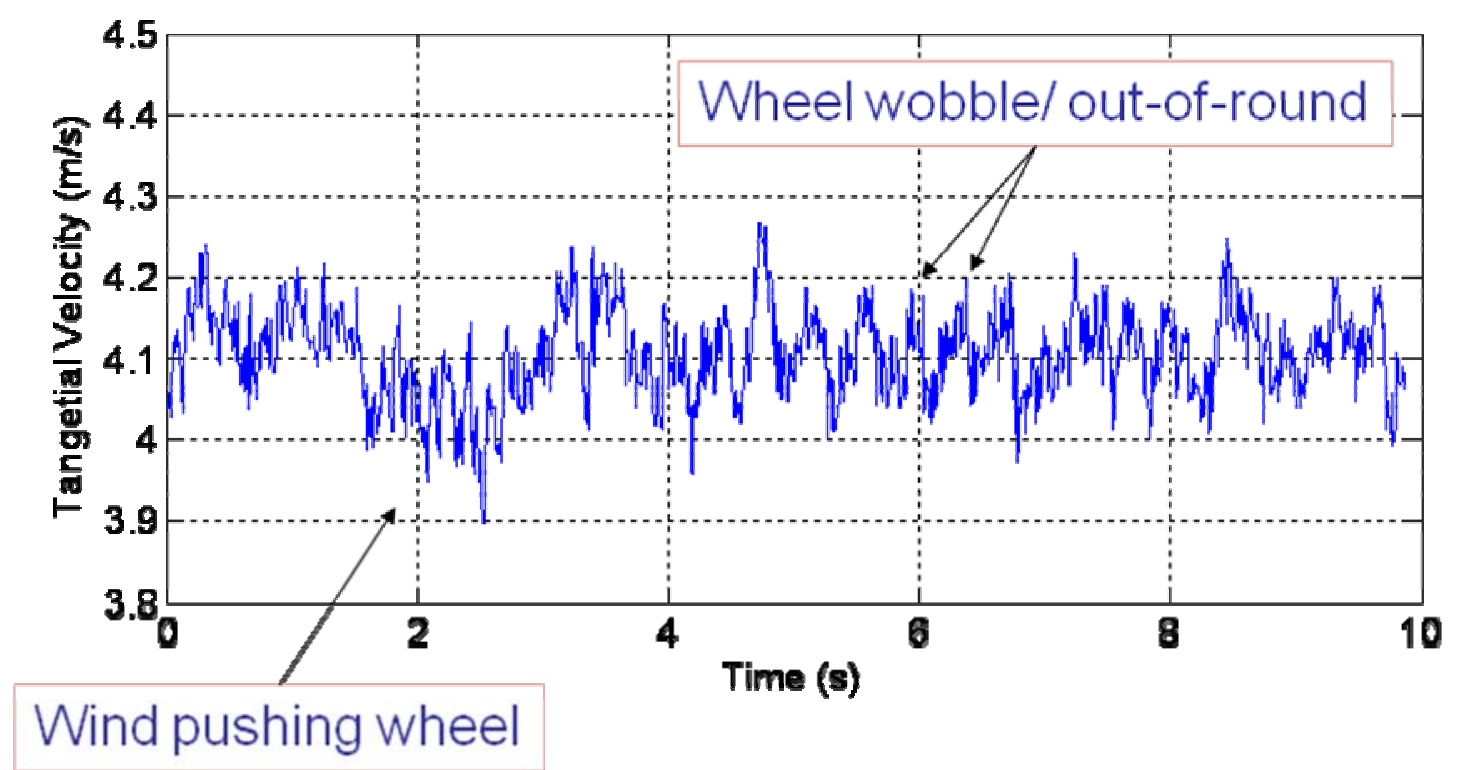

Figure 6. Velocity measurements of wheel obtained from 250-meters standoff range.

Velocity measurements were also made by scanning the lidar beam from above the normal incidence angle on the wheel to below the normal incidence angle, in order to obtain varying line-of-sight velocities and changing magnitude from positive to negative. Figure 7 shows a partial scan of the wheel velocity distribution above and below normal incidence. Discontinuities appearing on the velocity measurements are once again due to wind and wheel wobble. 


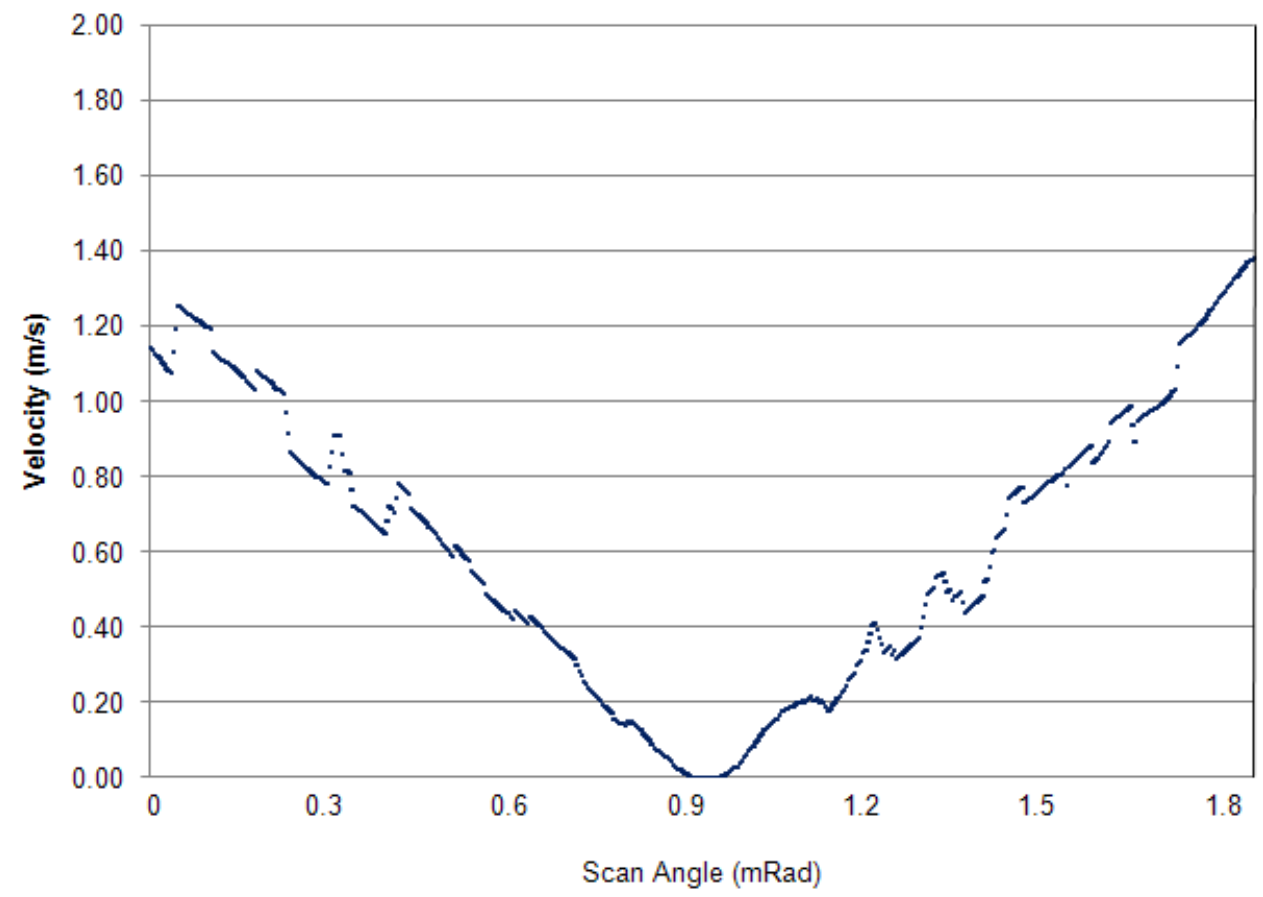

Figure 7. Line of sight velocity component scan of a rotating wheel. Zero velocity corresponds to normal incidence.

\section{SUMMARY}

A precision range and velocity lidar sensor is under development at NASA LaRC. Key features of this lidar are all-fiber design, narrow laser linewidth operation, excellent waveform modulation linearity, and eye-safe operation. The preliminary characterization tests indicate precisions of the order of 1-cm in range and 1- $\mathrm{cm} / \mathrm{sec}$ in velocity measurements. This lidar is currently being integrated into a relatively compact, rugged package for a series of tests from a helicopter platform. These helicopter tests are planned in support of NASA's Autonomous Landing and Hazard Avoidance Technology (ALHAT) project.

\section{REFERENCES}

1. Skolnik, M.I., "Introduction to Radar Systems” $2^{\text {nd }}$ ed., (McGraw-Hill Book Company, New York, 1980).

2. Saunders, W.K., "Post War Developments in Continuous-Wave and Frequency Modulated Radar,” IRE Trans., vol. ANE-8, pp.7-19, March 1961.

3. A.L. Kachelmyer, "Range-Doppler imaging: wave-forms and receiver design," Proc. SPIE, 999, 138-161, (1988).

4. Jelalian, A.V., "Laser Radar Systems,” (Artech House, Massachusetts, 1992).

5. Space Studies Board, National Research Council, New Frontiers in the Solar System - An Integrated Exploration Strategy, (National Academy Press, Washington, D.C., 2003). 
6. Golombek, M.P., Cook, R.A., et al, "Overview of the Mars Pathfinder Mission and Assessment of Landing Site Predictions,” Science Magazine, 278: 1743-1748, December 5, 1997.

7. Wong, E.C., et al.," Autonomous Guidance and Control Design for Hazard Avoidance and Safe Landing on Mars", AIAA Atmospheric Flight Mechanics Conference and Exhibit 5-8, 4619, Monterey, California, August 2002.

8. Pierrottet, D.F., et. al. "Development of an All-Fiber Coherent Laser Radar for Precision Range and Velocity Measurements”, Materials Research Society, San Francisco, California 2005.

9. Karlsson, C.J, et. al. "Linearization of the frequency sweep of a frequency-modulated continuous-wave semiconductor laser radar and the resulting ranging performance”, App. Opt., 38, 15, May 1999.

10. Pierrottet D.F., et. al. "Characterization of 3-D imaging lidar for hazard avoidance and autonomous landing on the Moon”, Proc. SPIE, Orlando, Florida, April 2007. 